\title{
ANÁLISE SOCIOECONÔMICA E CARACTERIZAÇÃO DOS SISTEMAS PESQUEIROS DA COMUNIDADE DE IGUAÍBA, MARANHÃO
}

Yago Bruno Silveira Nunes ${ }^{1,2^{*}}$; Thalline Santos Diniz ${ }^{2}$; Marina Bezerra Figueiredo ${ }^{1}$ ${ }^{1}$ Universidade Estadual do Maranhão, Laboratório de Biologia Pesqueira, São Luís, 65055-000, Maranhão, Brazil. ${ }^{2}$ Universidade Estadual do Maranhão, Centro de Ciências Agrárias, Graduando em Engenharia de Pesca.

* Autor correspondente: ybruno3098@gmail.com

\begin{abstract}
RESUMO
Este trabalho teve como objetivo identificar e caracterizar os aspectos socioeconômicos dos pescadores artesanais e a atividade pesqueira da comunidade de Iguaíba, localizada no município de Paço do Lumiar, Maranhão. A identificação de campo foi realizada por meio de visitas in loco. A principal técnica de coleta de dados foi a realização de entrevista semiestruturadas, contendo questões abertas e fechadas. Constatou-se que cerca de $95 \%$ dos pescadores entrevistados possuem o ensino fundamental incompleto. As pescarias são divididas entre o dono do barco e os pescadores, ou seja, $50 \%$ para o dono do barco e os outros $50 \%$ é dividido pelos outros pescadores. A comunidade pesqueira da Iguaíba é composta por pessoas que não dependem somente da pesca, mas sim de outras atividades produtivas, como: agricultura e manutenção de embarcações. Foram identificados oito sistemas de produção pesqueira na comunidade, sendo eles pedra, tainheira, malhão, manjuba, tarrafa, corvina, bandeirado e gozeira, em que a falta de investimentos acarreta na pouca autonomia das embarcações, nas condições de armazenamento dos organismos e na manutenção dos petrechos de pesca. A situação financeira das pessoas da região se caracteriza como relativamente baixa, já que dependem exclusivamente da pesca artesanal, da agricultura e de outras atividades lucrativas. Palavra-chave: Pesca artesanal, perfil socioeconômico, atividade pesqueira.
\end{abstract}

\section{SOCIOECONOMIC ANALYSIS AND CHARACTERIZATION OF THE FISHING SYSTEMS OF THE COMMUNITY OF IGUAÍBA, MARANHÃO}

\begin{abstract}
This work aimed to identify and characterize the socioeconomic aspects of artisanal fishermen and the fishery in the community of Iguaíba, located in the county of Paço do Lumiar, in the state of Maranhão. Field identification was carried out through on-site visits, as well as direct observations. The main data collection technique was semi-structured interview, containing open and closed questions. It was found that about $95 \%$ of the fishermen interviewed had incomplete elementary education. The fisheries are divided between the owner of the boat and the fishermen, with $50 \%$ for the owner of the boat and the other $50 \%$ divided by the other fishermen. The fishing community of Iguaíba is composed of people who do not depend only on fishing, but on other productive activities, such as agriculture and boat maintenance. Eight fishery production systems were identified in the community, such as stone, tainheira, malhão, manjuba, tarrafa, corvina, bandeirado and gozeira, in which lack of investment implies little vessel autonomy, storage conditions of organisms and maintenance of fishing gear. The financial situation of the people in the region is characterized as relatively low, as they depend exclusively on small-scale fisheries, agriculture and other earning activities.

Keywords: Artisanal fisheries, socioeconomic profile, fishing activity
\end{abstract}

\section{INTRODUÇÃO}

Os ambientes aquáticos sofrem diversos impactos que influenciam diretamente a fauna das regiões (Mourão et al., 2009). Sendo assim, sua importância científica, econômica e ambiental é elevada, visto que nesses ambientes ocorre um delicado equilíbrio ecológico devido à grande pressão antropogênica e da exploração de recursos naturais (Rodrigues, 2013).
O litoral Amazônico, que compreende os estados do Amapá, Pará e Maranhão, tem grande potencial para a pesca devido à sua grande quantidade de nutrientes originada a partir da matéria orgânica proveniente das florestas de mangue, que promove recursos para o desenvolvimento de muitos organismos aquáticos e, consequentemente, o aumento no potencial de produção dos recursos pesqueiros dessas regiões (Mourão, 2007; Clauzet et al., 2005; Figueiredo et al., 2014). 
Segundo Furtado Júnior et al. (2006), a exploração pesqueira artesanal no Brasil tem se caracterizado como uma das principais fontes de renda para diversas famílias que vivem da pesca e, dessa forma, essas comunidades tendem a conservar melhor a região, visando um melhor manejo no desenvolvimento da pesca (Dominguez et al., 2016; Santos et al., 2016).

De acordo com estudos feitos por Almeida et al. (2006), o Maranhão apresenta potencial para o desenvolvimento da atividade pesqueira, ainda que seja muitas vezes esquecida por parte dos governantes da região (Fernandes et al., 2015).

Para a realização da pesca artesanal, se faz necessário o uso de vários petrechos de pesca, que são os instrumentos empregados na captura de animais aquáticos, dentre eles: peixes, crustáceos, moluscos que podem variar de acordo com o instrumento de pesca (Monteles et al., 2010). No Maranhão, a pesca artesanal está representada por diversos tipos de petrechos, onde se destacam as armadilhas fixas, diferentes tipos de redes, espinhéis, puçá, além de redes de tapagem e zangaria (Araújo, 2008).

A comunidade de Iguaíba apresenta vários recursos pesqueiros de importância econômica, como estoques de pescadas amarela e outras espécies ainda inexploradas e pouco conhecidas. Por essa razão, se faz necessário estudos relacionados à atividade pesqueira nesta região.

Tendo em vista a lacuna de conhecimento sobre as atividades pesqueiras e também a necessidade de subsidiar informações que sejam úteis para o manejo pesqueiro da região, melhorando assim a economia e qualidade de vida das pessoas da comunidade, o presente estudo teve como objetivo identificar e caracterizar os aspectos socioeconômicos dos pescadores artesanais e a atividade pesqueira da comunidade de Iguaíba, localizada no município de Paço do Lumiar, Maranhão.

\section{MATERIAL E MÉTODOS}

\section{Caracterização da área estudada}

O litoral maranhenses possui aproximadamente $640 \mathrm{~km}$ de extensão (El-Robrini et al., 2015). A pesquisa foi realizada no município de Paço do Lumiar, mais precisamente na comunidade pesqueira da Iguaíba, localizada na porção nordeste da grande ilha de São Luís (Figura 1).

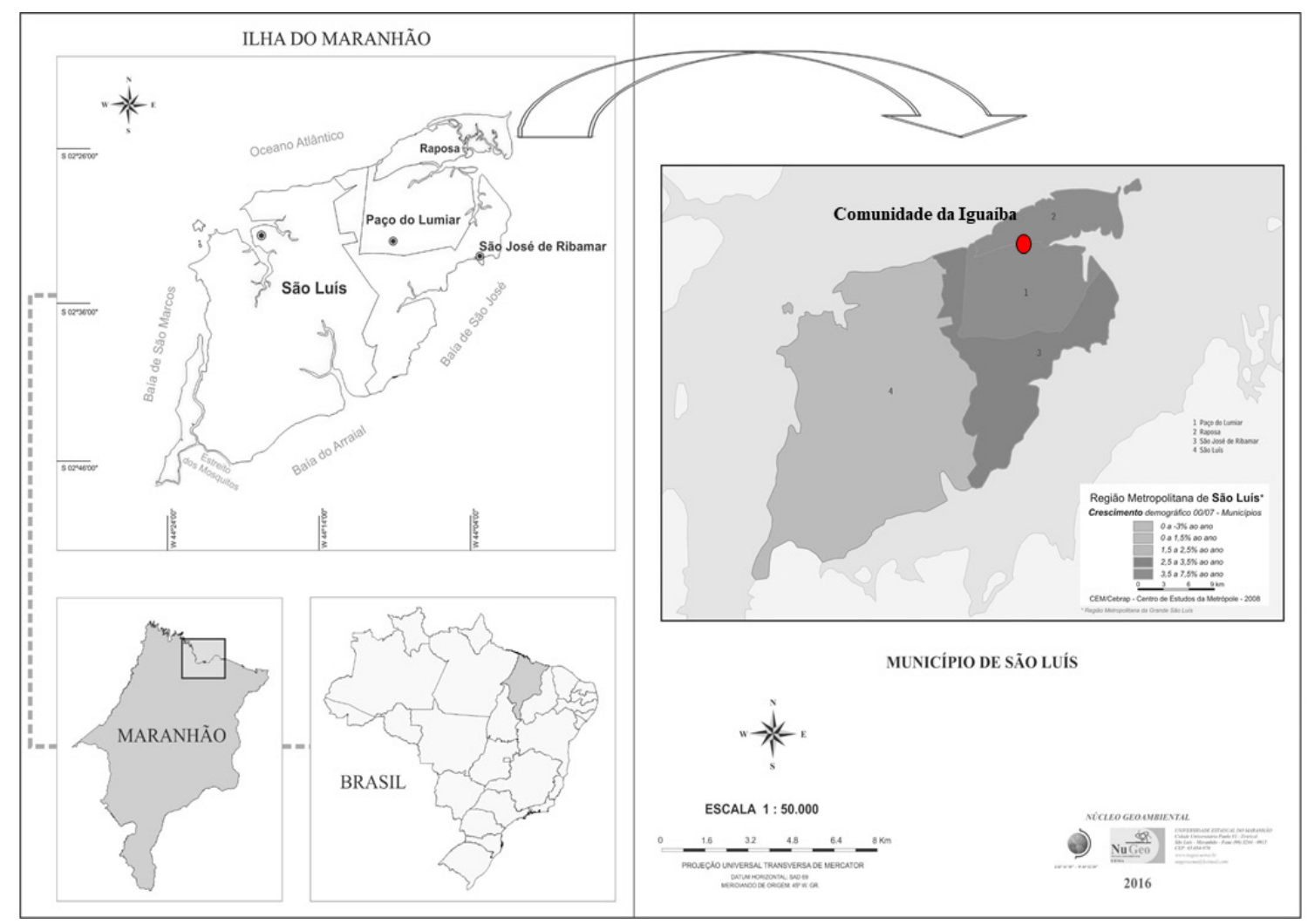

Figura 1. Mapa da área de estudo, comunidade pesqueira de Iguaíba - MA. 
O município possui extensas áreas verdes ainda não ocupadas por atividades humanas, bem como uma rede hidrográfica privilegiada e extensos manguezais. Tendo isso como base, diversas atividades são desenvolvidas no município, principalmente o extrativismo, a pesca e agricultura familiar (Prefeitura de Paço do Lumiar, 2017).

\section{Coleta dos dados}

Para a caracterização dos Sistemas Pesqueiros da comunidade estudada, foram determinados assuntos envolvendo as questões Social, Ecológica, Econômica, Tecnológica e Manejo Pesqueiro. Somado ao levantamento dessas informações, foi realizada uma identificação em campo através de visitas in loco. Como complemento dos dados, foram desenvolvidas pesquisas de revisão bibliográfica, tais como em artigos científicos, dissertações, teses, publicações em revistas e sites nacionais, com o intuito de melhorar o fundamento das informações.

A principal técnica de coleta de dados foi a realização entrevistas semi-estruturadas, contendo questões abertas e fechadas. Para cada sistema foi elaborada uma ficha de caracterização similar ao que foi utilizado por Almeida (2008), onde foram usados as seguintes perguntas: nome da espécie-alvo; aspectos biológicos da espécie-alvo; área de atuação dos pesqueiros; tecnologia e atividade pesqueira; número de pescadores; produção média; época de safra; cadeia de comercialização; preço; renda; complemento de renda; relação de trabalho e partilha da produção; qualidade de vida; grau de escolaridade; organização social; conflitos na comunidade, e manejo. Por fim, as informações foram processadas com o auxílio do programa Excel 2010.

\section{RESULTADOS E DISCUSSÃO}

\section{Características socioeconômicas}

Foram entrevistados um total de 20 pescadores na comunidade de Iguaíba. As casas dos pescadores entrevistados são próprias e de alvenaria, possuem água de poço e saneamento básico. A comunidade não possui hospital, apenas um posto de saúde que atende a população local. Cerca de $95 \%$ dos pescadores entrevistados possuem o ensino fundamental incompleto, $4 \%$ possuem ensino fundamental completo e $1 \%$ possuem ensino médio incompleto. A falta de escolaridade adequada entre as comunidades ribeirinhas ainda é uma realidade do nosso país, tal resultado também foi encontrado por Vaz et al. (2017), em duas comunidades localizadas no estado do Pará. Nossos resultados sobre a baixa escolaridade encontrada entre os pescadores da comunidade de Iguaíba foram similares à realidade encontrada no município da Raposa como retratado em estudo proposto por Santos et al. (2011).

Béné (2006) conceitua pobreza como sendo a falta de acesso a necessidade básica, como por exemplo, a saúde, educação, água potável, entre outros serviços. Tais problemas foram constantemente relatados pelos moradores da comunidade de Iguaíba. Situação equivalente à que foi identificada por Monteles et al. (2009) na comunidade das marisqueiras do município da Raposa, qual as pessoas da região não tinham tanto acesso a esses recursos básicos.

A comunidade estudada relatou que estoques pesqueiros das principais espécies comerciais também se encontram em diminuição, sendo concordante com os resultados estatísticos apresentados pela FAO (2015) em vários locais do planeta. Pois, o esforço de pesca exercido para suprir a demanda gerada pelo crescimento populacional tem sido muito intenso, acarretando na estagnação e diminuição populacional de algumas espécies de peixes (Allison \& Ellis, 2001). Tal fato influencia na diminuição da renda dos pescadores, tendo em vista que precisam dividir seu lucro com o dono e os demais membros da embarcação ( $50 \%$ para o dono do barco e os outros $50 \%$ é dividido pelos outros pescadores).

A época de safra dos pescados alvo geralmente corresponde ao período chuvoso (janeiro a julho), porque muitas espécies se aproximam da costa. Além disso, este período é mais seguro para a navegação os pescadores, aumentando o tráfego de embarcações de pesca. Enquanto isso, os meses de estiagem apresentam fortes ventos, o que reduz o potencial pesqueiro nesse período, pois os peixes se afastam da costa. Tais resultados são corroborados por Corrêa et al. (2012) em seus estudos no município de Coari, localizado na região amazônica, em que o autor identificou que o período de safra também ocorre durante os meses de chuva.

A cadeia de comercialização do pescado na comunidade em estudo incide na venda para os atravessadores, podendo chegar até $\mathrm{R} \$ 20,00$ o quilo dos peixes, e assim são vendidos em feiras por um preço mais elevado. Garcez \& SánchezBotero (2005), observaram que o escoamento da produção de pescados das comunidades artesanais do Rio Grande do Sul também necessita da figura do atravessador, sendo similar ao da Iguaíba. Contudo, é importante salientar que Monteles et al. (2009), ao estudar a comunidade da Raposa, percebeu que $8 \%$ da produção de moluscos extraída da região é 
comercializada para atravessadores, o que significa dizer que possuem outras fontes de escoamento para sua produção, o que facilitaria a renda familiar.

Os pescadores dessa região desempenham outras funções no período de defeso, como conserto e manutenção de barcos e confecção de artes de pesca, assim não comprometem o sustento de sua família. Além disso, a pouca remuneração pode influenciar na mudança nos tamanhos dos petrechos de pesca (Almeida, 2008; Zacardi et al., 2017), já que os pescadores não conseguem adquirir os materiais necessários para a confecção dos mesmos, dificuldade que também é observada na comunidade de Iguaíba.

A comunidade pesqueira possui um Sindicato dos pescadores e aquicultores de Iguaíba, sendo que a maioria dos pescadores não estão associados a esta instituição, o que gera conflitos na região, pois somente os pescadores sindicalizados têm direitos aos benefícios. No estudo realizado por Nascimento et al. (2016) em Suape (PE) foi verificado que o associativismo propicia maior confiabilidade dos associados na colônia ( $53,3 \%$ dos entrevistados), porém os resultados encontrados na comunidade pesqueira de Iguaíba foram contrários e demonstraram que a colônia local possui dificuldades em conseguir recursos em função da falta de confiabilidade dos membros não associados em se associarem.

\section{Caracterização dos sistemas de pesca e embarcações}

Foram identificados oito sistemas de produção pesqueira na comunidade de Iguaíba, sendo fundamentais para o sustento das famílias.

Os sistemas pesqueiros, bem como as embarcações em Iguaíba são diversificados, podendo ser de pequeno e médio porte (Tabela 1). Almeida et al. (2006) e Nascimento et al. (2016) apresentam em seus estudos que as embarcações utilizadas no litoral maranhense são feitas de fibra de vidro, variando de 5 a 7 metros e possuem vela triangular latina.

O sistema Pedra tem como espécie alvo o peixe pedra (Genyatremus luteus), mas tem como fauna acompanhante diversos organismos, tais como: tainha, pescadinha, bagre, corvina, entre outros. Esta espécie reside em águas com concentração de sal menor que 30, ou seja, estuários e lagunas, ambientes com cascalhos, areia e pedras (Almeida, 2008).

A arte de pesca utilizada para a captura dessa espécie é a linha com anzóis de números 14 ou 15 , semelhante com a arte de pesca encontrado por Zacardi et al. (2016), em estudo realizado no município Calçoene no estado do Amapá. Devido seu hábito alimentar, utiliza-se iscas vivas e frescas, como por exemplo camarões e pequenos peixes. As embarcações usadas nesse sistema são barcos de pequeno porte de tamanho de 5,5 a $6,5 \mathrm{~m}$, com motor de 5 a $9,5 \mathrm{Hp}$, respectivamente. Na embarcação vão 2 a 3 pessoas por pescaria.

No sistema Tainheira, o principal recurso capturado é a tainha (Mugil curema), apresentando como fauna acompanhante o peixe pedra (Genyatremus luteus) e a pescada gó (Macrodon ancylodon). Os barcos utilizados apresentam 7 metros de comprimento e motores de $11 \mathrm{Hp}$. As artes de pesca comumente usadas pelos pescadores são a rede de arrasto e o espinhel, bem com os estudos de Monteles et al. (2010), que identificaram essa arte de pesca capturando espécies de fundo.

A tainha (M. curema), por sua vez, está distribuída em águas tropicais e subtropicais marinhas em várias regiões do globo, podendo ser encontrada em zonas costeiras, estuarinas e marinhas (Seckendorff \& Azevedo, 2007; Oliveira, 2010). A tainha alimenta-se principalmente de algas microscópicas, pequenos organismos planctônicos e detritos, e pode atingir em sua grande maioria cerca de $30 \mathrm{~cm}$ de comprimento (Seckendorff \& Azevedo, 2007).

O sistema de pesca do tipo Malhão, tem como espécie alvo a pescada amarela (Cynoscion acoupa). Para a captura de C. acoupa, os pescadores da comunidade de Iguaíba necessitam de uma rede de emalhar atrelados a barcos com estrutura de 7 metros e motor de $11 \mathrm{Hp}$. Tais resultados também foram encontrados por Paz et al. (2011), que observaram na comunidade de Vila do Conde, região do Pará, que os pescadores utilizam redes de emalhar para a captura da pescada-branca (Plagioscion squamosissimus) e o peixe sarda (Pellona castelnaeana), assim como embarcações motorizadas de pequeno e médio porte. Em estudos realizados por Monteles et al. (2010), foi possível identificar que, no município de Humberto de Campus e Primeira Cruz, se utilizam: currais, linha e redes de enseadas para a captura de pescadas.

Segundo Mourão et al. (2009) o processo de comercialização da pescada amarela (C. acoupa) começa com a pesca artesanal em barcos com pouca tecnologia, e, em seguida, uma parte do produto é vendida após o desembarque no porto. A carne da pescada amarela é considerada nobre, por isso seu valor no mercado é muito alto podendo ser entre $\mathrm{R} \$$ 160,00 a R\$ 185,00 por kg (MOURÃO et al., 2009). Sua vesícula gasosa possui elevado valor comercial e exportada para o exterior, pois é utilizada na indústria 
Tabela 1. Caracterização dos sistemas pesqueiros da comunidade de Iguaíba - MA.

\begin{tabular}{|c|c|c|c|c|c|c|}
\hline SISTEMA & FROTA & ARTE & $\begin{array}{l}\text { RECURSO } \\
\text { PRINCIPAL }\end{array}$ & AMBIENTE & $\begin{array}{l}\text { RELAÇÃO DE } \\
\text { TRABALHO }\end{array}$ & RENDA \\
\hline PEDRA & $\begin{array}{l}\text { Barcos com } \\
5.5 \text { metros } \\
\text { com motor de } \\
5 \mathrm{Hp}, \text { Barco } \\
\text { com } 6,5 \text { me- } \\
\text { tros e motor de } \\
9,5 \text { Hp e Sem } \\
\text { embarcação }\end{array}$ & $\begin{array}{l}\text { Linha de } \\
\text { mão }\end{array}$ & $\begin{array}{l}\text { Peixe pedra } \\
\text { (Genyatremus } \\
\text { luteus), corvina } \\
\text { (Cynoscion micro- } \\
\text { lepidotus), pescada } \\
\text { gó (Macrodon } \\
\text { ancylodon) e ban- } \\
\text { deirado (B. bagre) }\end{array}$ & $\begin{array}{l}\text { Estuário e } \\
\text { praia }\end{array}$ & Familiar & Média \\
\hline TAINHEIRA & $\begin{array}{l}\text { Barco com } 7 \\
\text { metros com } \\
11 \mathrm{Hp}\end{array}$ & $\begin{array}{l}\text { Rede de } \\
\text { arrasto e } \\
\text { espinhel }\end{array}$ & $\begin{array}{l}\text { Tainha (Mugil } \\
\text { curema), peixe } \\
\text { pedra }(G \text {. luteus) } \\
\text { e pescada gó (M. } \\
\text { ancylodon) }\end{array}$ & $\begin{array}{l}\text { Estuário e } \\
\text { praia }\end{array}$ & $\begin{array}{l}\text { Familiar/Ami- } \\
\text { gos }\end{array}$ & Média \\
\hline MALHÃO & $\begin{array}{l}\text { Barcos de } 7 \\
\text { metros com } \\
11 \mathrm{Hp} \\
\end{array}$ & $\begin{array}{l}\text { Rede de } \\
\text { emalhar }\end{array}$ & $\begin{array}{l}\text { Pescada amarela } \\
\text { (Cynoscion acou- } \\
\text { pa) }\end{array}$ & Costa & Familiar & Média \\
\hline MANJUBA & $\begin{array}{l}\text { Barcos com } \\
5.5 \text { metros } \\
\text { com motor de } \\
5 \text { Hp e Barco } \\
\text { com } 6,5 \text { me- } \\
\text { tros e motor de } \\
9,5 \mathrm{Hp} \\
\end{array}$ & $\begin{array}{l}\text { Rede de } \\
\text { arrasto }\end{array}$ & $\begin{array}{l}\text { Manjuba (Ancho- } \\
\text { viella sp), pescada } \\
\text { gó (Macrodon } \\
\text { ancylodon) e ban- } \\
\text { deirado (Bagre } \\
\text { bagre) }\end{array}$ & $\begin{array}{l}\text { Estuário e } \\
\text { costa }\end{array}$ & Familiar & Média \\
\hline TARRAFA & \begin{tabular}{|l|} 
Sem embar- \\
cação ou com \\
canoa com \\
motor \\
\end{tabular} & \begin{tabular}{|l|} 
Tarrafa e \\
armadilha
\end{tabular} & $\begin{array}{l}\text { Camarão piticaia } \\
\text { (Xiphopenaeus } \\
\text { kroyeri) }\end{array}$ & Estuário & Familiar & Média \\
\hline CORVINA & $\begin{array}{l}\text { Barco com } 6 \\
\text { metros e mo- } \\
\text { tor de } 9 \mathrm{Hp}\end{array}$ & $\begin{array}{l}\text { Rede de } \\
\text { emalhar }\end{array}$ & $\begin{array}{l}\text { Corvina (Cynos- } \\
\text { cion microlepido- } \\
\text { tus) e peixe pedra } \\
(\text { G. luteus) }\end{array}$ & Estuário & Familiar & Média \\
\hline BANDEIRADO & \begin{tabular}{|l} 
Barcos com \\
5 metros com \\
motor de $5 \mathrm{Hp}$ \\
\end{tabular} & $\begin{array}{l}\text { Rede de } \\
\text { emalhe }\end{array}$ & $\begin{array}{l}\text { Bandeirado (Bagre } \\
\text { bagre) e peixes } \\
\text { pequenos }\end{array}$ & $\begin{array}{l}\text { Estuário e } \\
\text { costa }\end{array}$ & Familiar & Média \\
\hline GOZEIRA & $\begin{array}{l}\text { Barcos com } \\
7 \text { metros com } \\
11 \mathrm{Hp} \mathrm{e} \mathrm{Barco} \\
\text { com } 6,5 \text { me- } \\
\text { tros e motor de } \\
9,5 \mathrm{Hp}\end{array}$ & $\begin{array}{l}\text { Rede de } \\
\text { emalhe }\end{array}$ & $\begin{array}{l}\text { Pescada gó (Ma- } \\
\text { crodon ancylodon) } \\
\text { e bandeirado ( } \mathrm{Ba}- \\
\text { gre bagre) }\end{array}$ & $\begin{array}{l}\text { Estuário e } \\
\text { Costa }\end{array}$ & Familiar & Média \\
\hline
\end{tabular}

farmacológica (Ramires et al., 2003; Piorski et al., 2010).

No sistema Manjuba são utilizados barcos de 5,5 metros, com motor de $5 \mathrm{Hp}$ e Barco com 6,5 metros e motor de 9,5 Hp. O petrecho utilizado é a rede de arrasto, com medidas de $30 \times 30,30 \mathrm{x}$ 35. A espécie alvo é a manjuba (Anchoviella sp), mas também podem ser encontrados a pescada gó (Macrodon ancylodon) e o bandeirado (Bagre bagre). Esta atividade é realizada no município da Raposa localizado na costa do estado do Maranhão conforme estudo feitos por Santos et al. (2011).

O sistema Tarrafa pode ou não usar embarcação, e a mais utilizada é a canoa com motor. O petrecho utilizado é a tarrafa, uma pequena rede de $20 \mathrm{~cm}$ x $20 \mathrm{~cm}$ que utiliza pesos de chumbo na parte inferior denominada de saco, onde são retidos os peixes. É destinada a peixes variados, além do camarão piticaia (Xiphopenaeus kroyeri). A captura de camarão, principalmente, por redes ou tarrafas também foram 
encontrados no estudo de Freire \& Silva (2008), com os camarões Macrobrachium amazonicum e $M$. rosembergii na região de Bragantina no Pará.

A arte de pesca rede de emalhar também é muito utilizada na comunidade de Iguaíba, em que são utilizados barcos com 6 metros e motor de $9 \mathrm{Hp}$. São redes de $40 \mathrm{~cm} \times 40 \mathrm{~cm}$, que ficam no fundo, meia água e superfície. As espécies alvos são a corvina (Cynoscion microlepidotus) e o peixe pedra (G. luteus).

Barcos com 5 metros com motor de $5 \mathrm{Hp}$, também utilizam rede de emalhar de $50-60 \mathrm{~m}$. A espécie alvo é o bandeirado (Bagre bagre), peixe de ambiente estuarino que se alimenta de caranguejos (Almeida et al., 2008). O bandeirado é conservado em caixas de isopor com gelo. Tais resultados são parecidos com aqueles encontrados por Espírito-Santo \& Isaac (2012) no município de Bragança, na região do Pará, onde pescadores capturam o peixe bandeirado com embarcações relativamente pequenas.

Referente à caracterização do sistema Gozeira, foi detectado o uso de redes de emalhar, cujo principal recurso é a Pescada Gó (Macrodon ancylodon), e como a fauna acompanhante é possível encontrar o bandeirado (B. bagre). As embarcações na comunidade de estudo, tem barcos com 6 metros com motor de 9,5 Hp e barcos com 7 metros com motor de $11 \mathrm{Hp}$.

A pescada-gó ( $M$. ancylodon), também conhecida pelos nomes de pescadinha-gó, corvina de boca-mole e pescada-foguete, é um peixe demersal marinho de vasta distribuição geográfica, ocorrendo tanto em águas tropicais como em águas subtropicais, podendo os indivíduos desta espécie ocorrer em ambientes estuarinos (Castro et al., 1998; Freire et al., 2011). Segundo Fonseca \& Castro (2000), a pescada-gó é amplamente comercializada no Maranhão, principalmente pelo seu alto valor de comércio e ótimo sabor da carne.

\section{CONCLUSÃO}

A comunidade pesqueira da Iguaíba é composta por pessoas que não dependem somente da pesca, mas também de outras atividades produtivas, como, por exemplo agricultura e manutenção de embarcações. A atividade pesqueira da comunidade apresentou uma variedade de sistemas pesqueiros, que operam desde a região estuarina até a zona costeira, utilizando artes de pesca artesanais e com pouca seletividade. Por fim, cada sistema utiliza embarcações de pequeno porte, com pouca tecnologia para comunicação, levando a uma autonomia reduzida.

\section{REFERÊNCIAS BIBLIOGRÁFICAS}

ALLISON, E.H.; ELLIS, F. 2001. The livelihoods approach and management of small-scale fisheries. Elsevier Science Ltd. All rights reserved. Marine Policy 25: 377-388.

ALMEIDA, Z.S. 2008. Os recursos pesqueiros marinhos e estuarinos do Maranhão: biologia, tecnologia, socioeconômicas, estado da arte e manejo. [Dissertação], Universidade Federal do Pará, Belém.

ALMEIDA, Z.S.; FERREIRA, D.S.C.; NAHUM, V.J.I. 2006. Classificação e evolução das embarcações maranhenses. Boletim do Laboratório de Hidrobiologia, 19:31-40.

ARAÚJO, C.M.E. 2008. Fauna acompanhante do sistema de produção pesqueira pescada amarela (Cynoscion acoupa - pisces: Scianidae, Lacepéde 1802) desembarcada na praia do Araçagy área do litoral da Ilha do Maranhão, Brasil: subsídios para sua conservação. [Pós-graduação], Universidade Federal do Maranhão, São Luís.

BÉNÉ, C. 2006. Small-scale fisheries: assessing their contribution to rural livelihoods in developing countries. FAO Fisheries Circular. No. 1008. Rome, 46p.

CASTRO, A.C.L. 1998. Idade e crescimento de Plagioscion squamosissimus (Heckel, 1840) (Acanthopterygii, Sciaenidae) do reservatório de Barra Bonita-SP, através da estrutura dos otólitos. Acta Scientiarum 20 (2):179-184.

CLAUZET, M.; RAMIRES, M.; BARRELLA, W. 2005. Pesca artesanal e conhecimento local de duas populações caiçaras (enseada do mar virado e barra do una) no litoral de São Paulo, Brasil. A linguagem da Ciência. v 4, maio.

CORRÊA, M.A.A.; KAHN, J.R.; FREITAS, C.E.C. 2012. A Pesca no Município de Coari, Estado do Amazonas, Brasil. Revista Brasileira de Engenharia de Pesca 6(2): I-XII.

DOMINGUEZ, P.S.; ZEINEDDINE, G.C.; ROTUNDO, M.M.; BARRELLA, W.; RAMIRES, M. 2016. A pesca artesanal no arquipélago de Fernando de Noronha (PE). Boletim do Instituto de Pesca, São Paulo, 42(1): 241-251.

EL-ROBRINI, M.; MARQUES, J. V.; SILVA, M. A. M. A.; EL-ROBRINI, M. H.; FEITOSA, A. C.; TAROUCO, J. E. F.; SANTOS, J. H. S.; VIANA, J. R. 2015. Erosão e progradação do litotal brasileiro/ Maranhão. Brasil: Ministério do Meio Ambiente, p.87-130. 
ESPÍRITO-SANTO, R.V.; ISAAC, V.J. 2012. Desembarques da Pesca de Pequena Escala no Município de Bragança - PA, Brasil: Esforço e Produção. Boletim do Laboratório de Hidrobiologia, 25(1):31-48.

FAO. 2015. Estadísticas de pesca y acuicultura. Food And agriculture Organization Of the United Nations. Rome, 107p. ISBN 978-92-5-009987-3.

FERNANDES, S.C.P.; BENTES, A.B.; PEREIRA, L.J.G.; NASCIMENTO, M.S.; SILVA, B.B. 2015. Variação temporal da captura comercial do peixe pedra, Genyatremus luteus, desembarcado em um pólo pesqueiro da costa norte do BRASIL PENÍNSULA de Ajuruteua-Bragança-PA. Boletim do Instituto de Pesca, São Paulo, 41(1): 173 - 182.

FIGUEIREDO, M.B.; CARVALHO NETA, R.N.F.; NUNES, J.L.S.; ALMEIDA, Z.S. 2014. Feeding habits of Macrodon ancylodon (Actinopterygii, Sciaenidae) in northeast, Brazil. Revista de Biología Marina y Oceanografía, 49 (3): 559-566, DOI 10.4067/S0718-19572014000300012

FONSECA, F.A.L.; CASTRO, A.C.L. 2000. Dinâmica Da Nutrição da Pescada gó Macrodon ancylodon (Bloch \& Schneider, 1801) (Teleostei: Sciaenidae), Na Costa Do Estado Do Maranhão. Boletim do Laboratório de Hidrobiologia, 13 (1).

FREIRE, J.L.; SILVA, B.B.; SOUZA, A.S. 2011. Aspectos Econômicos e Higiênico-Sanitários da Comercialização do Pescado no Município de Bragança (PA). Biota Amazônia. Macapá, 1 (2): 17-28.

FREIRE, J.L.; SILVA, B.B. 2008. Aspectos sócio-ambientais das pescarias de camarões Dulcíolas (Macrobrachium amazonicum Heller, 1862 e Macrobrachium rosenbergii de Man, 1879) (Decápoda, Palaemonidae) na região Bragantina - Pará - Brasil. Boletim do Laboratório de Hidrobiologia, 21:51-62.

FURTADO JÚNIOR, I.; TAVARES, M.C.S.; BRITO, C.S.F. 2006. Estatísticas das produções de pescado estuarino e marítimo do estado do Pará e políticas pesqueiras. Bol. Mus. Para. Emílio Goeldi. Ciências Humanas, Belém, 1 (2): 95-111, maio-ago.

GARCEZ, D.S.; SÁNCHEZ-BOTERO, J.I. 2005. Comunidades de pescadores artesanais no Estado do Rio Grande do Sul, Brasil. Atlântica, Rio Grande, 27 (1): 17-29.

MONTELES, J.S.; CASTRO, T.C.S.; VIANA, D.C.P.; CONCEIÇÃO, F.S.; FRANÇA, V.L.; FUNO, I.C.S.A. 2009. Percepção Socio-Ambiental das
Marisqueiras no Município de Raposa, Maranhão, Brasil. Revista Brasileira de Engenharia de Pesca, 4(2): 34-45.

MONTELES, J.S.; FUNO, I.C.A.; CASTRO, A.C.L. 2010. Caracterização da pesca artesanal nos municípios de Humberto de Campos e Primeira Cruz - Maranhão. Boletim do Laboratório de Hidrobiologia, 23:65-74.

MOURÃO, K.R.M.; FRÉDOU, F.L.; ESPÍRITO SANTO, R.V.; ALMEIDA, M.C.; SILVA, B.B.; Thierry FRÉDOU, T.; ISAAC, V. 2009. Sistema de produção pesqueira pescada amarela - Cynoscion acoupa Lacèpede (1802): um estudo de caso no litoral nordeste do Pará - Brasil. Boletim do Instituto de Pesca, São Paulo, 35(3): 497 - 511.

MOURÃO, K. R. M. 2007. Sistema de produção pesqueira pescada amarela (Cynoscion acoupa Lacèpede, 1802) e Serra (Scomberomorus brasiliensis Collette, Russo \& Zavalla-Camin, 1978) no litoral nordeste do Estado do Pará. [Dissertação], Universidade Federal do Pará, 130 p., Belém.

NASCIMENTO, M.J.S.; COELHO-FILHO, P.A.; CASTRO, N.A. 2016. Aspectos sócio-econômicos da pesca artesanal em Suape, cabo de Santo Agostinho, Pernambuco (Brasil). Revista Brasileira de Engenharia de Pesca, 9(1):65-76.

OLIVEIRA, M. R. 2010. Biologia reprodutiva da tainha, Mugil curema Valenciennes, 1836 (Osteichthyes: Mugilidae) nas águas costeiras do Rio Grande do Norte. [Dissertação], Universidade Federal do Rio Grande do Norte, Natal.

PAZ, A. C.; FRÉDOU, F. L.; FRÉDOU, T. 2011. Caracterização da atividade pesqueira em Vila do Conde (Barcarena, Pará), no estuário amazônico. Boletim do Museu Paraense Emílio Goeldi. Ciências Humanas, 6 (2): 307-318.

PIORSKI, N.M.; NUNES, J.L.S.; ALMEIDA, Z.S. 2010. Records of the smalleye smoothhound Mustelus higmani Springer \& Lowe, 1963 (Chondrichthyes: Triakidae) from Maranhão - Brazil. Boletim do Laboratório de Hidrobiologia, 23:75-78.

PREFEITURA DE PAÇO DO LUMIAR. Paço do Lumiar: Histórico. Disponível em: http://www. pacodolumiar.ma.gov.br/pagina/paco-do-lumiar/1. Acesso em: 05 nov de 2017.

RAMIRES, M; BARELLA, W; ESTEVES, A. M. 2003. Caracterização da pesca artesanal e o conhecimento pesqueiro local no valedo ribeira e litoral Sul de São Paulo. Revista Cecilliana. 0378-1844. 
RODRIGUES, D. 2013. Ecologia trófica das principais espécies de peixes do Rio Paraíba do Sul na área de influência do aproveitamento hidrelétrico simplício durante o período pré-represamento. [Pósgraduação], Juiz de Fora.

SANTOS, P.R.S.; EINHARDT, A.C.M.C.; VELASCO, G. 2016. A pesca artesanal da miragaia (Pogonias cromis, Sciaenidae) no estuário da Lagoa dos Patos, Brasil. Boletim do Instituto de Pesca, São Paulo, 42(1): 89-101.

SANTOS, P.V.C.J.; ALMEIDA-FUNO, I.C.S.; PIGA, F.G.; FRANÇA, V.L.; TORRES, S.A.; MELO, C.D.P. 2011. Perfil Socioeconômico de Pescadores do Município da Raposa, Estado Do Maranhão. Revista Brasileira de Engenharia de Pesca, 6(1): I-XIV.

SECKENDORFF, R. W. V; AZEVEDO, V. G. 2007. Abordagem histórica da pesca da tainha Mugil platunus e do parati Mugil curema (Perciformes: Mugilidae) no litoral norte do estado de São Paulo. Série Relatórios Técnicos, São Paulo, 28: 1-8.

VAZ, E.M.; RABELO, Y.G.S.; CORRÊA, J.M.S.; ZACARDI, D.M. 2017. A pesca artesanal no lago Maicá: aspectos socioeconômicos e estrutura operacional. Biota Amazônia. Macapá, 7 (4): 6-12.

ZACARDI, D.M.; SILVA, G.S.; VAZ, E.M.; SILVA, L.M.A. 2016. Estudo Dos Aspectos Sociais E Técnicos Da Atividade Pesqueira No Município De Calçoene, Amapá, Extremo Norte Do Brasil. Revista Brasileira de Engenharia de Pesca, 9(2): 52-68.

ZACARDI, D.M.; SARAIVA, M.L.; VAZ, E.M. 2017. Caracterização da pesca artesanal praticada nos lagos Mapiri e Papucu às margens do rio Tapajós, Santarém, Pará. Rev. Bras. Eng. Pesca 10(1): 31-43. 